\title{
UGT74D1 Is a Novel Auxin Glycosyltransferase from Arabidopsis thaliana
}

\author{
Shang-Hui Jin ${ }^{1,2}$, Xin-Mei Ma ${ }^{1,2}$, Ping Han ${ }^{1,2}$, Bo Wang ${ }^{1,2}$, Yan-Guo Sun ${ }^{1,2}$, Gui-Zhi Zhang ${ }^{1,2}$, Yan-Jie Li ${ }^{1,2}$, \\ Bing-Kai Hou ${ }^{1,2 *}$
}

1 The Key Lab of Plant Cell Engineering and Germplasm Innovation, Education Ministry of China, Jinan, Shandong Province, P. R. China, 2 School of Life Science, Shandong University, Jinan, Shandong Province, P. R. China

\begin{abstract}
Auxin is one type of phytohormones that plays important roles in nearly all aspects of plant growth and developmental processes. The glycosylation of auxins is considered to be an essential mechanism to control the level of active auxins. Thus, the identification of auxin glycosyltransferases is of great significance for further understanding the auxin regulation. In this study, we biochemically screened the group L of Arabidopsis thaliana glycosyltransferase superfamily for enzymatic activity toward auxins. UGT74D1 was identified to be a novel auxin glycosyltransferase. Through HPLC and LC-MS analysis of reaction products in vitro by testing eight substrates including auxins and other compounds, we found that UGT74D1 had a strong glucosylating activity toward indole-3-butyric acid [IBA], indole-3-propionic acid [IPA], indole-3-acetic acid [IAA] and naphthaleneacetic acid [NAA], catalyzing them to form corresponding glucose esters. Biochemical characterization showed that this enzyme had a maximum activity in HEPES buffer at $\mathrm{pH} 6.0$ and $37^{\circ} \mathrm{C}$. In addition, the enzymatic activity analysis of crude protein and the IBA metabolite analysis from transgenic Arabidopsis plants overexpressing UGT74D1 gene were also carried out. Experimental results indicated that over-production of the UGT74D1 in plants indeed led to increased level of the glucose conjugate of IBA. Moreover, UGT74D1 overexpression lines displayed curling leaf phenotype, suggesting a physiological role of UGT74D1 in affecting the activity of auxins. Our current data provide a new target gene for further genetic studies to understand the auxin regulation by glycosylation in plants.
\end{abstract}

Citation: Jin S-H, Ma X-M, Han P, Wang B, Sun Y-G, et al. (2013) UGT74D1 Is a Novel Auxin Glycosyltransferase from Arabidopsis thaliana. PLoS ONE 8(4): e61705. doi:10.1371/journal.pone.0061705

Editor: Gloria Muday, Wake Forest University, United States of America

Received October 26, 2012; Accepted March 12, 2013; Published April 16, 2013

Copyright: ( 2013 Jin et al. This is an open-access article distributed under the terms of the Creative Commons Attribution License, which permits unrestricted use, distribution, and reproduction in any medium, provided the original author and source are credited.

Funding: This research was supported by grants from the National Natural Science Foundation of China (No. 90917006 and No. 30971543 to B.K.H.). The funders had no role in study design, data collection and analysis, decision to publish, or preparation of the manuscript.

Competing Interests: The authors have declared that no competing interests exist.

*E-mail: bkhou@sdu.edu.cn

\section{Introduction}

Auxin is the first discovered phytohormone and is well-known for its regulatory role in virtually all aspects of plant growth and development, such as general root and shoot architecture, organ initiation and patterning, cell division and differentiation [1-3], plant responses to biotic and abiotic stresses, etc [4-5]. Auxins belong to chemically diverse compounds, most of which have an aromatic system such as indole, phenyl or naphthalene ring with a side chain containing a carboxyl group attached. The hormone is known to exist as the free acid or in conjugation with a wide variety of compounds such as amino acids, peptides, and sugars [6].

Indole-3-acetic acid (IAA) is the most abundant auxin natively generated in plants and its in vivo role has been examined extensively. However, there are other native endogenous auxins in plants. Indole-3-butyric acid (IBA) has been identified in a number of plant species such as maize (Zea mays), pea (Pisum sativum) and Arabidopsis, comprising approximately $25 \%$ to $30 \%$ of the total free auxin pool in Arabidopsis seedlings [7]. It is reported that the concentration of free IBA is comparable to the level of free IAA in a number of plants [8]. As for IBA in vivo functions, many studies support the idea that IBA only functions as a precursor of IAA. For example, IBA can be converted to IAA in a process similar to fatty acid $\beta$-oxidation and the IBA-to-IAA conversion enzymes have been identified [9-14]. In addition, many Arabidopsis mutants unable to convert IBA to active IAA have reduced root growth sensitivity to IBA, but normal sensitivity to IAA, suggesting that IBA is an essential auxin precursor rather than active auxin [13-17]. However, it is also argued in other literatures that IBA may have activity independent of IAA. For example, the activity of IBA can affect lateral root induction, adventitious root initiation, elongation of roots, shoots, and hypocotyls [18-20], as well as the induction of auxin-responsive genes [21-22]. In a recent report, the increase of free IBA, but not IAA level in UGT74E2OE plants indicated that the shoot morphogenesis might be directly affected by IBA rather than its conversion to IAA [23]. This partial independence of both auxins was further supported by accounted differences in IAA and IBA polar transport $[18,19,24,25]$.

The hormonal homeostasis is defined as "the maintenance of a steady state concentration of the hormones in the receptive tissue appropriate to any fixed environmental condition" [6]. Plants use several mechanisms to control the level of endogenous auxins. Despite the regulation of synthesis and degradation of these phytohormones, plants may store auxins in the form of conjugates [26]. IAA and IBA can be conjugated via amide linkage to amino acids such as aspartate and by ester linkages to glucose or myo- 
inositol [27-29]. The level of conjugated forms of IAA and IBA may be higher than their free forms and are considered to be inactive storage and/or transport forms of the hormone [29-30]. Their enzymatic release to free forms is an important part of auxin metabolism. For IBA, its ester conjugates dominate over amide forms of IBA. Moreover, IBA conjugates are more easily hydrolyzed and more slowly transported in different plant systems, perhaps leaving more phytohormones at the plant base in comparison with conjugates of IAA [9,29,31]. In addition, certain IBA conjugates are very active in bioassays $[9,27]$. The formation and hydrolysis of auxin conjugates is developmentally regulated and varies significantly among plant tissues [32].

Glucose conjugates of both IBA and IAA have been identified in plants, including Arabidopsis [9,31,33]. Glycosyltransferases catalyze the addition of sugar to auxins. Since glycosylation can alter many properties of the aglycones in respect to their bioactivity, solubility, as well as their cellular localization, glycosylation is considered as an important regulatory mechanism for the cellular homeostasis and activity of phytohormones [34-35]. So far, several auxin glycosyltransferases have been identified from plants. iaglu is the first auxin glycosyltransferase gene identified from Zea mays [36]. UGT84B1 and UGT74E2 were then identified from Arabidopsis and chemically demonstrated that they have high activity toward auxins, particularly toward IAA and IBA, respectively [23,37]. Three related enzymes (UGT84B2, UGT75B1, and UGT75B2) were also identified with trace activities [37]. When overexpressing UGT84B1 or UGT74E2 in Arabidopsis, a clear disturbance in auxin homoestasis and obvious growth defects were observed [23,38]. These findings suggest that auxin glycosyltranferases are important players for auxin activity and plant development. In addition, the existence of multiple glycosyltransferases toward the same type of phytohormone in one plant species may implicate a synergistic effect of multiple glycosyltransferase members beneficial for plant evolution and adaptation.

Our research interest is to screen phytohormone-related glycosyltransferases from Arabidopsis. Till now, 120 UDP-glycosyltransferase (UGT) have been identified and classified into 14 groups in the Arabidopsis genome [39-40]. Among them, group L becomes our first target because several hormone-related UGTs, including UGT84B1 and UGT74E2, were identified from this group (Figure 1). In our screening, UGT74D1 was identified to be a novel auxin glycosyltransferase, but we cannot exclude the possible activity for other members. We provide in this study solid evidence to show the enzyme activity and biochemical characterization of UGT74D1. Moreover, a metabolites analysis of auxin glucosyl-conjugates and phenotypic analysis for the UGT74D1 overexpressing transgenic plants were carried out as well.

\section{Materials and Methods}

\section{Chemicals}

Most of the substrates used in this study were purchased from Sigma-Aldrich (St. Louis, MO USA). UDP-Glucose was purchased from Meryer (Shanghai, China). Glutathione-coupled Sepharose $4 \mathrm{~B}$ beads and reduced form glutathione were obtained from Amersham Pharmacia (Piscataway, NJ USA). Restriction enzymes, ligation enzymes and PrimeSTAR HS DNA Ploymerase were purchased from TaKaRa (Shiga, Japan).

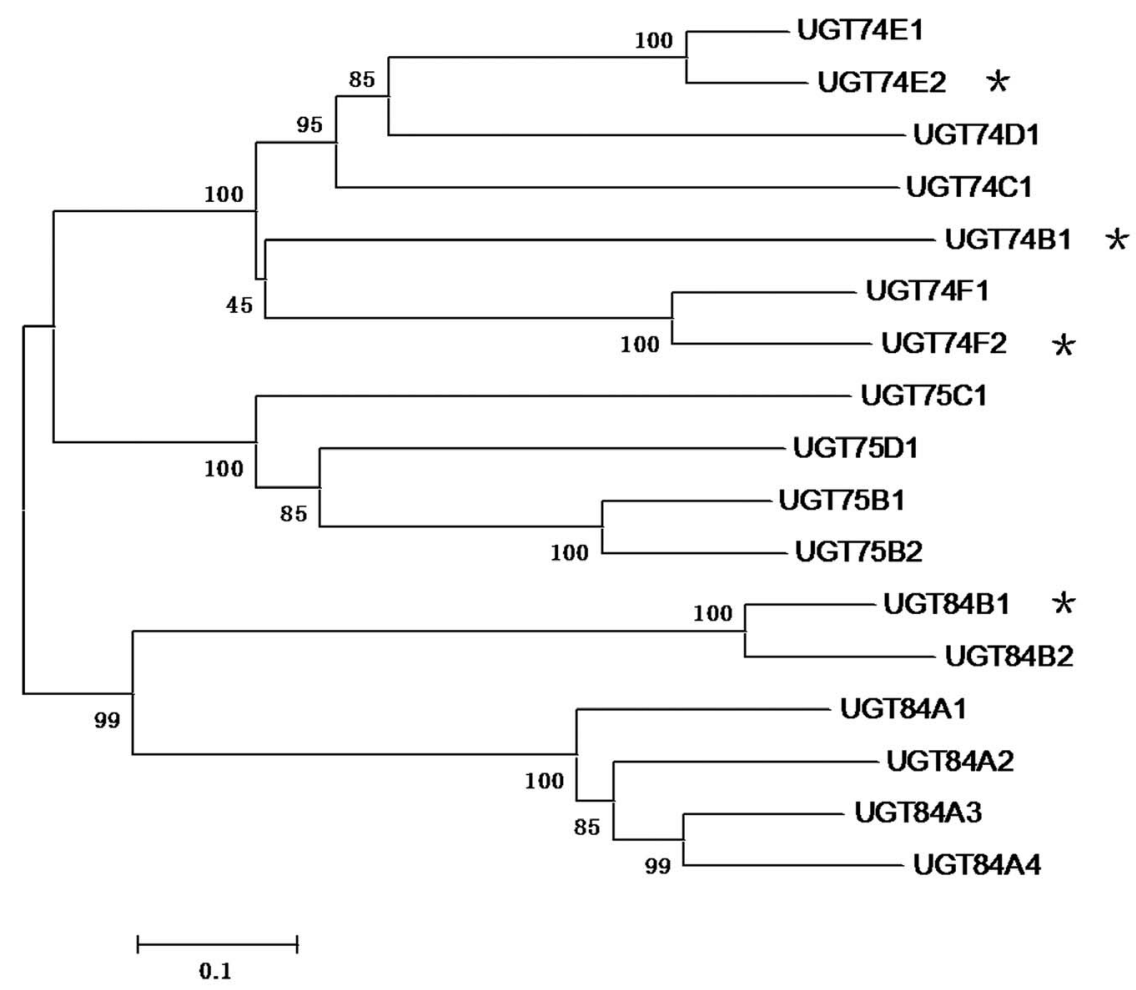

Figure 1. Phylogenetic relationship of the group L glycosyltransferases from Arabidopsis thaliana. The phylogenetic tree of Arabidopsis UGTs is adopted from the previous report [40]. Bootstrap values are indicated above the nodes. The glycosyltransferase sequences were retrieved from Carbohydrate-active enzymes database (http://www.cazy.org/GT1_eukaryota.html) and NCBI database. The asterisks indicate those glycosyltransferases with confirmed enzymatic activities toward phytohormone related compounds.

doi:10.1371/journal.pone.0061705.g001 


\section{kDa $\quad M \quad$ GST $\quad$ 74D1}

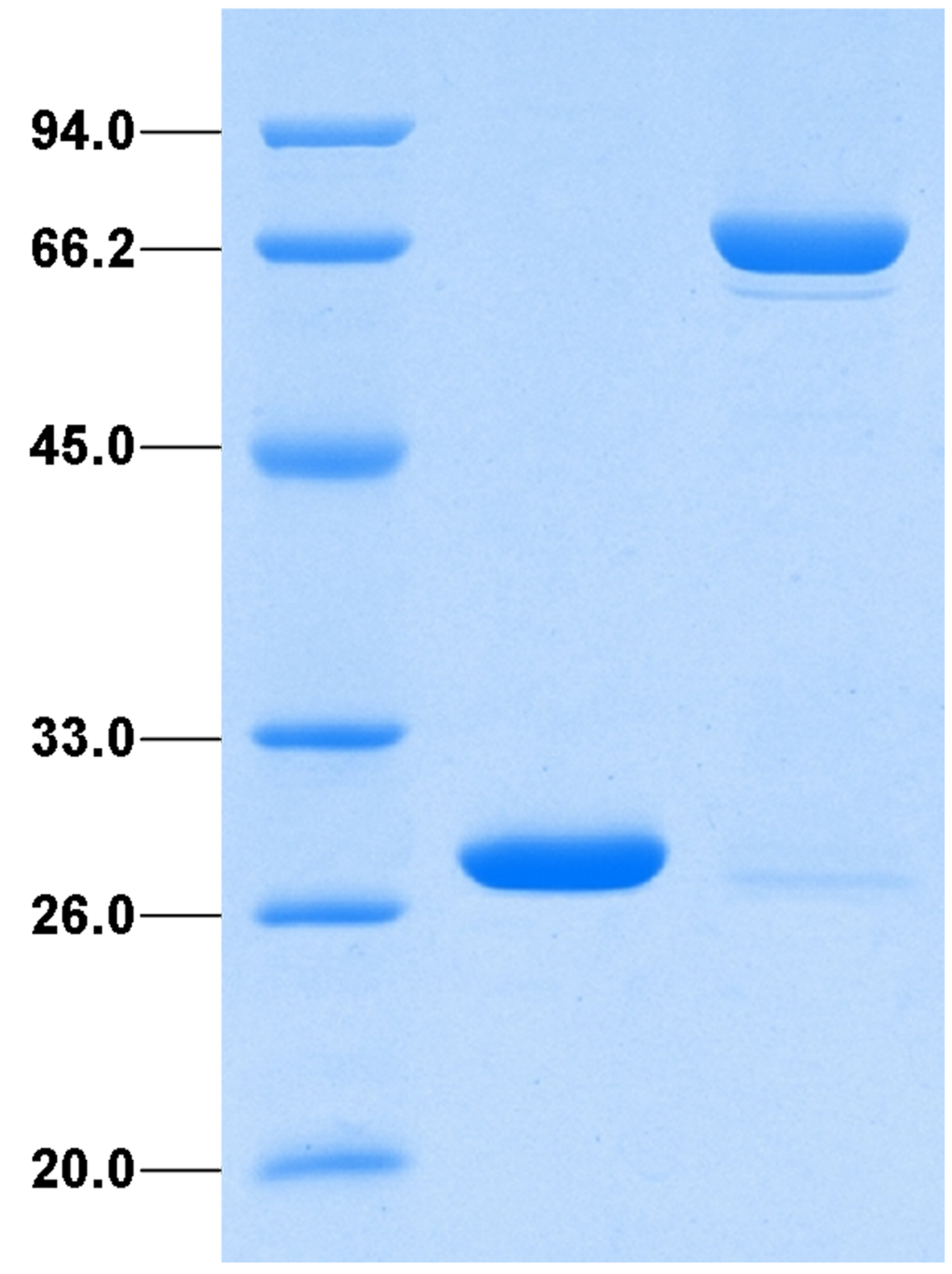

Figure 2. SDS-PAGE analysis of the recombinant GST-UGT74D1 fusion protein. Proteins were purified from E.coli, analyzed on a $12 \%$ (w/v) polyacrylamide gel and visualized with Coomassie Brilliant Blue staining. M: protein molecular weight marker; GST: glutathione-s-transferase; 74D1: fusion protein.

doi:10.1371/journal.pone.0061705.g002

\section{Cloning, Plasmid Construction and Sequence Analysis of UGT74D1}

Standard DNA manipulation techniques were used. Full-length cDNA of UGT74D1(At2g31750) was amplified from Arabidopsis by reverse transcription-PCR (RT-PCR) with a pair of primers, UGT74D1-a: 5'-CGCCATATGGGAGAGAAAGCGAAAGG$3^{\prime}$ and UGT74D1-b: 5'-CGGCTCGAGTTACGTCACAATTTTAGC-3', which contain restriction sites at 5' terminals for $\mathcal{N d e I}$ and $X h_{0} \mathrm{I}$, respectively. The PGR product was cloned by recombination into pBluescriptSK. In order to obtain a prokaryotic expression vector with suitable and multiple restriction sites, pGEX-2T vector was modified according to methods described by Zhang and co-authors [41] with a slight modification. The modified pGEX-2T vector has the multiple clone sites BamHI, NdeI, NotI, SphI, NcoI, SalI, SacI, XhoI, HindIII, EcoRI and is designated as pGEX-3H. UGT74D1cDNA was subcloned from pBluescript SK plasmid into pGEX-3H between the sites of $\mathcal{N d e I}$ and $X h o \mathrm{I}$ to obtain the expression plasmid of GST-UGT74D1 fusion protein.

The phylogeny of 17 gene which were in the $\mathrm{L}$ group of Arabidopsis family 1 glycosyltransferases were obtained from the alignments using ClustalX 2 and Neighbor-Joining trees constructed with bootstrap sampling of 1000 replications using MEGA 4.0 programs. The Arabidopsis UGT sequences used in the phylogenetic tree were obtained from the Carbohydrate-active enzymes database (http://www.cazy.org/GT1_eukaryota.html) and the NCBI database. 

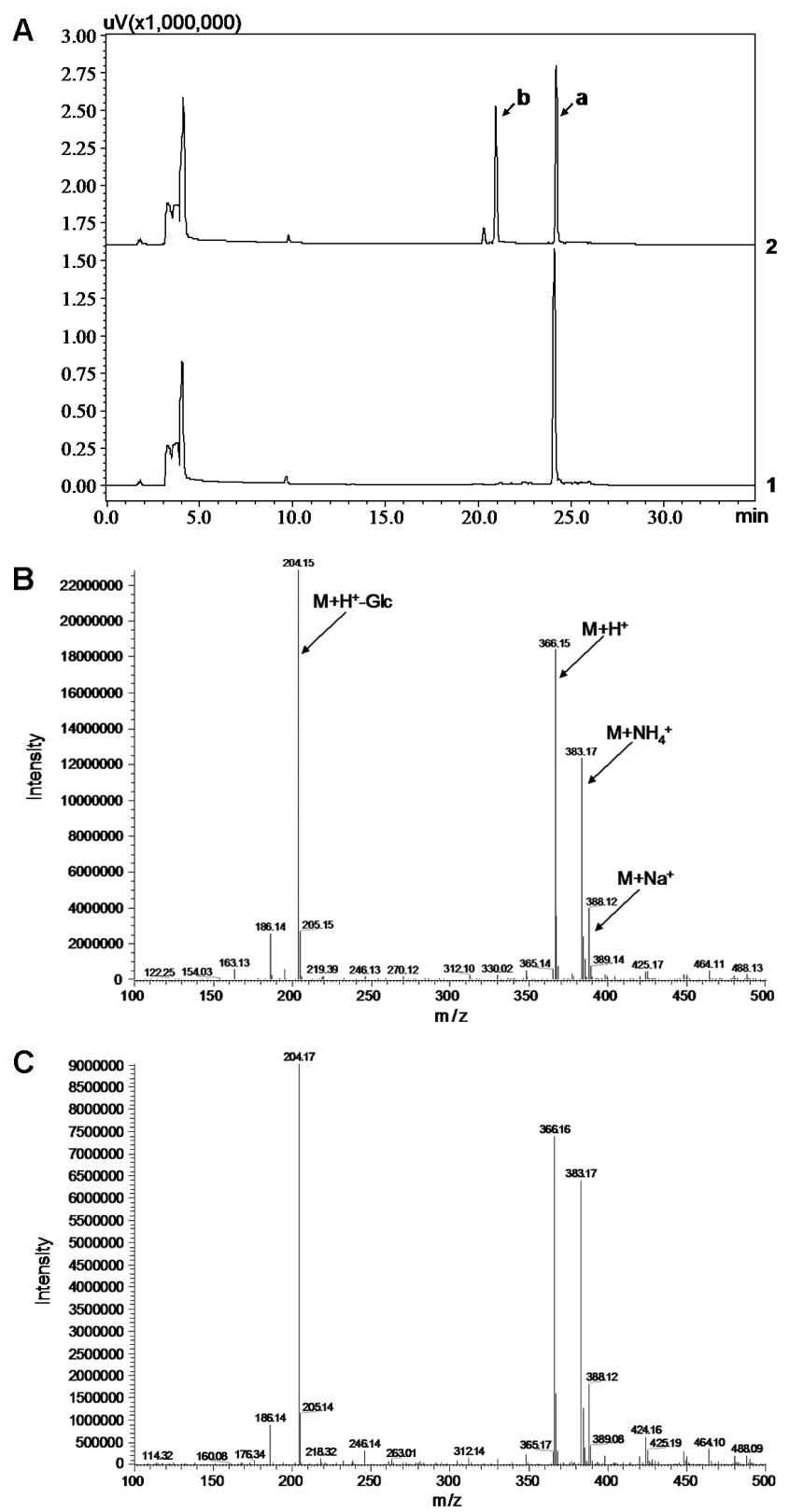

D

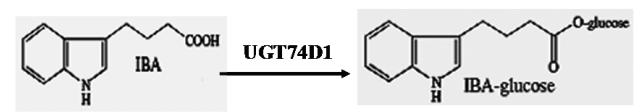

Figure 3. HPLC and LC-MS analysis of reaction product from IBA. (A) HPLC analysis. 1: the reaction was added with GST protein as control. 2: the reaction was added with UGT74D1 fusion protein and a new peak (peak b) was produced. Peak "a" represents the substrate IBA. (B) LC-MS analysis of peak b. (C) LC-MS analysis of IBA glucose conjugates produced by the catalysis of UGT74E2 which was used as positive control in this research. (D) Proposed enzymes and biosynthetic pathway for the synthesis of IBA-glucose ester from the aglycone IBA. doi:10.1371/journal.pone.0061705.g003

\section{Protein Putification and Enzyme Activity Assay}

Escherichia coli strain XL1-Blue carrying the expression plasmid of GST-UGT74D1 fusion construct was used to produce the fusion protein. Soluble recombinant protein was induced and purified according to the methods described by Hou et al. [42].
Protein concentration of the eluted fractions was determined with Coomassie Protein Assay Reagent (Thermo Scientific) using bovine serum albumin as reference. The purified recombinant fusion protein was also analyzed by SDS-PAGE following the methods described by Sambrook et al [43].

The glycosyltransferase activity assay was carried out following the conditions described by Tognetti and co-workers with modifications [23]. The assay mix $(100 \mu \mathrm{l})$ contained $2 \mathrm{ug}$ of purified UGT74Dl fusion protein, $5 \mathrm{mM}$ UDP-glucose, $1 \mathrm{mM}$

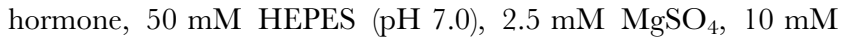
$\mathrm{KCl}$ and $14.4 \mathrm{mM}$ 2-mercaptoethanol. The reactions were carried out at $37^{\circ} \mathrm{C}$ for $3 \mathrm{~h}$ and then stopped by the addition of $10 \mu \mathrm{l}$ of trichloroacetic acid $(240 \mathrm{mg} / \mathrm{ml})$, quick-frozen, and stored at $-20^{\circ} \mathrm{C}$ before reverse-phase HPLC analysis.

\section{HPLC and LC/MS Analysis}

$20 \mu \mathrm{l}$ of each sample was loaded by means of a auto sampler SIL-20A (Shimadzu HPLC system equipped with the diode array detector SPD-M20A, the SIL-20A, the commuications bas module CBM-20A, the degasser DGU-20A3 and the workstation LC solution), onto a $5 \mu \mathrm{m}$ C18 column $(150 \times 4.6 \mathrm{~mm}$; Welch, Ultimate). A linear gradient with increasing methanol (solvent A) against distilles $\mathrm{H}_{2} \mathrm{O}$ (solvent $\mathrm{B}$ ) at a flow rate of $1 \mathrm{ml} / \mathrm{min}$ over $30 \mathrm{~min}$ was used to separate the glucose conjugates from their aglycones. Both solutions contained $0.01 \% \mathrm{H}_{3} \mathrm{PO}_{4}$. Each peak on the chromatogram was monitored between 190 and $430 \mathrm{~nm}$.

The HPLC conditions were described in the following: IAA, $\lambda_{\text {detection }}=210 \mathrm{~nm}, 10 \%-48 \%$ solvent A; ICA, IPA, IBA, and NAA, $\lambda_{\text {detection }}=280 \mathrm{~nm}, 10 \%-70 \%$ solvent A; 2,4-dichlorophenoxyacetic acid and picloram, $\lambda_{\text {detection }}=287 \mathrm{~nm}, 10 \%-100 \%$ solvent A.

The products of auxin conjugates synthesized by recombinant UGT74D1 were further confirmed by the LG-MS system (Thermo Scientific) including the Surveyor autosampler and MS pump (Thermo-Finnigan, San Jose, CA, USA). The methods and mobile phases were similar to HPLC condition except that $0.01 \%$ acetic acid instead of $0.01 \% \mathrm{H}_{3} \mathrm{PO}_{4}$. The mass spectrometer operated in a positive electrospray ionization mode with $30 \mathrm{eV}$ and a probe voltage of $3.0 \mathrm{kV}$. The temperature was set to $350^{\circ} \mathrm{C}$. The data acquisition and analysis were performed with Xcalibur software (version 2.0.6).

Table 1. Specific activity of UGT74D1 toward auxins and related substrates.

\begin{tabular}{ll}
\hline Substrates & Specific activity (nkat/mg protein) \\
\hline ICA & $0.17 \pm 0.03$ \\
IAA & $1.25 \pm 0.01$ \\
IPA & $1.85 \pm 0.01$ \\
IBA & $2.17 \pm 0.05$ \\
NAA & $1.15 \pm 0.01$ \\
$2,4-D$ & $0.32 \pm 0.08$ \\
Picloram & ND \\
\hline
\end{tabular}

Note: The assay mix $(100 \mu \mathrm{l})$ contained 2 ug of purified UGT74D1 fusion protein, $5 \mathrm{mM}$ UDP-glucose, $1 \mathrm{mM}$ hormone, $50 \mathrm{mM}$ HEPES (pH 7.0), $2.5 \mathrm{mM}$ $\mathrm{MgSO}_{4}, 10 \mathrm{mM} \mathrm{KCl}$ and $14.4 \mathrm{mM}$ 2-mercaptoethanol. The reactions were carried out at $37^{\circ} \mathrm{C}$ for $3 \mathrm{~h}$. The results represent the means $\pm S . D$ from three independent measurements. The specific enzyme activity was defined as nmol of substrates converted into glucose conjugates per second (nanokatal, nkat) by $1 \mathrm{mg}$ of protein.

doi:10.1371/journal.pone.0061705.t001 

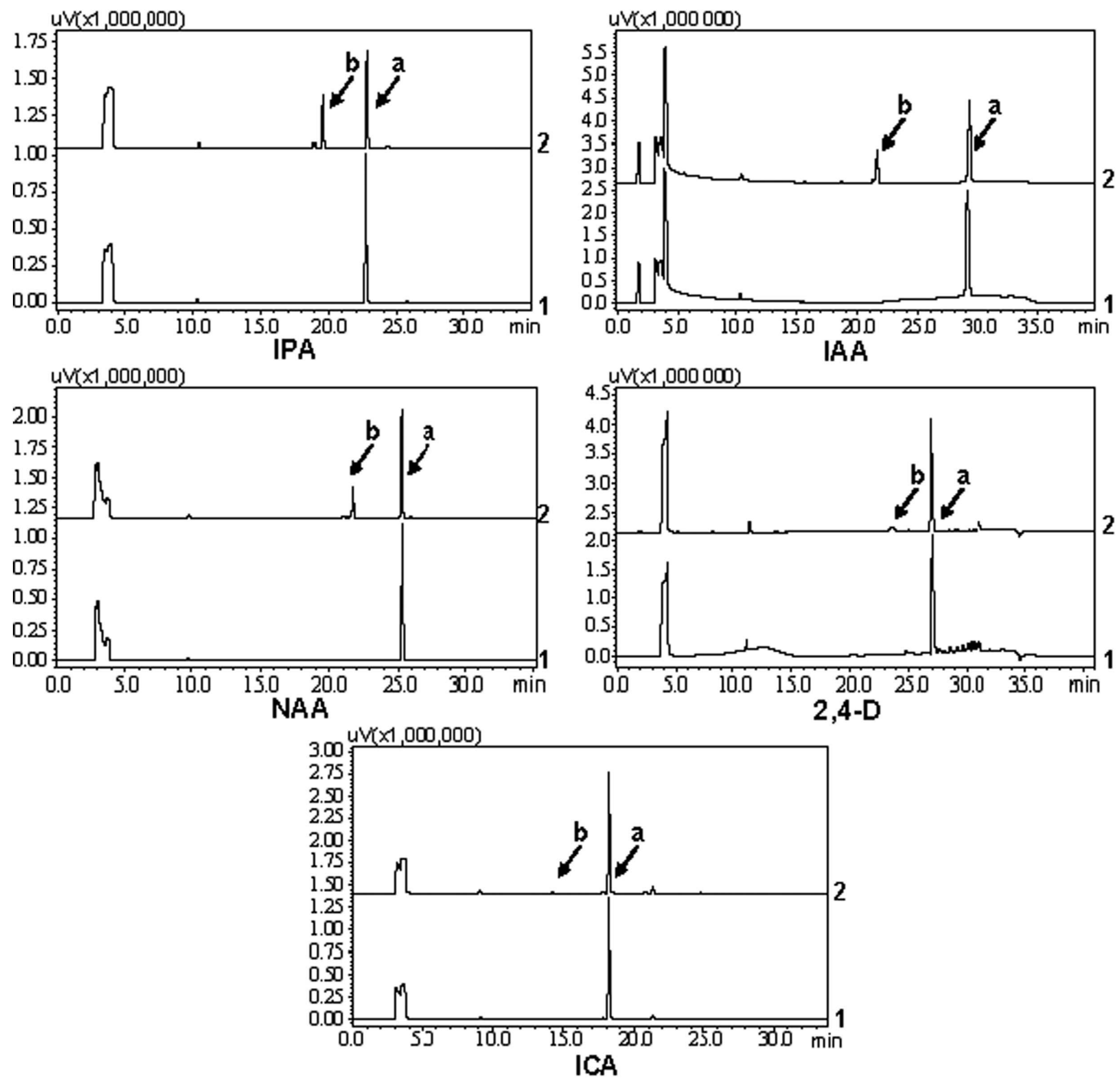

Figure 4. HPLC analysis of reaction products from other auxins. 1: the reaction was added with GST protein as control. 2: the reaction was added with UGT74D1 fusion protein. Peak "a" represents the auxin substrates. Peak "b" represents the reaction products.

doi:10.1371/journal.pone.0061705.g004

Factors Affecting the Activity of Recombinant UGT74D1

Because UGT74D1 has the highest enzyme activity toward IBA, we choose the IBA in this study as substrate for analyzing the factors affecting the enzyme activity. The calculation of enzyme activity was based on the reduction of peak area of the substrate IBA before and after reaction. Factors tested include temperature, buffer and $\mathrm{pH}$. All the reaction mix $(100 \mu \mathrm{l})$ contained $0.2 \mathrm{ug}$ of recombinant UGT74D1, $5 \mathrm{mM}$ UDP-glucose, $1 \mathrm{mM}$ IBA, $2.5 \mathrm{mM} \mathrm{MgSO}_{4}, 10 \mathrm{mM} \mathrm{KCl}, 14.4 \mathrm{mM}$ 2-mercaptoethanol. For the temperature test, $50 \mathrm{mM}$ HEPES (pH7.0) was added and the reactions were performed at four different temperature values $\left(20^{\circ} \mathrm{C}, 30^{\circ} \mathrm{C}, 37^{\circ} \mathrm{C}\right.$ and $\left.45^{\circ} \mathrm{C}\right)$. For the buffer and $\mathrm{pH}$ test, $50 \mathrm{mM}$ Tris buffer ( $\mathrm{pH} 6.0-9.0), 50 \mathrm{mM}$ HEPES buffer (pH 5.0-9.0),
$50 \mathrm{mM}$ MES buffer (pH 5.0-9.0) or $50 \mathrm{mM}$ phosphate buffer ( $\mathrm{pH}$ 6.0-9.0) was added and the reactions were performed at $37^{\circ} \mathrm{C}$. All the reactions were carried out for $30 \mathrm{~min}$ and then stopped by adding $10 \mu \mathrm{l}$ trichloroacetic acid $(240 \mathrm{mg} / \mathrm{ml})$, quickfrozen, and stored at $-20^{\circ} \mathrm{C}$ before reverse-phase HPLC analysis.

Assays of Glycosyltransferase Activity and Glucosylated Metabolites of Auxin in Transgenic Plants

The full-length cDNA of the UGT74D1 gene was subcloned from the pBluescriptSK into the plant overexpression vector pBI121 and replaced the glucuronidase (GUS) gene. The overexpression construct was transferred into Agrobacterium tumefaciens GV3101 and then transformed into Arabidopsis (Col-0) via 

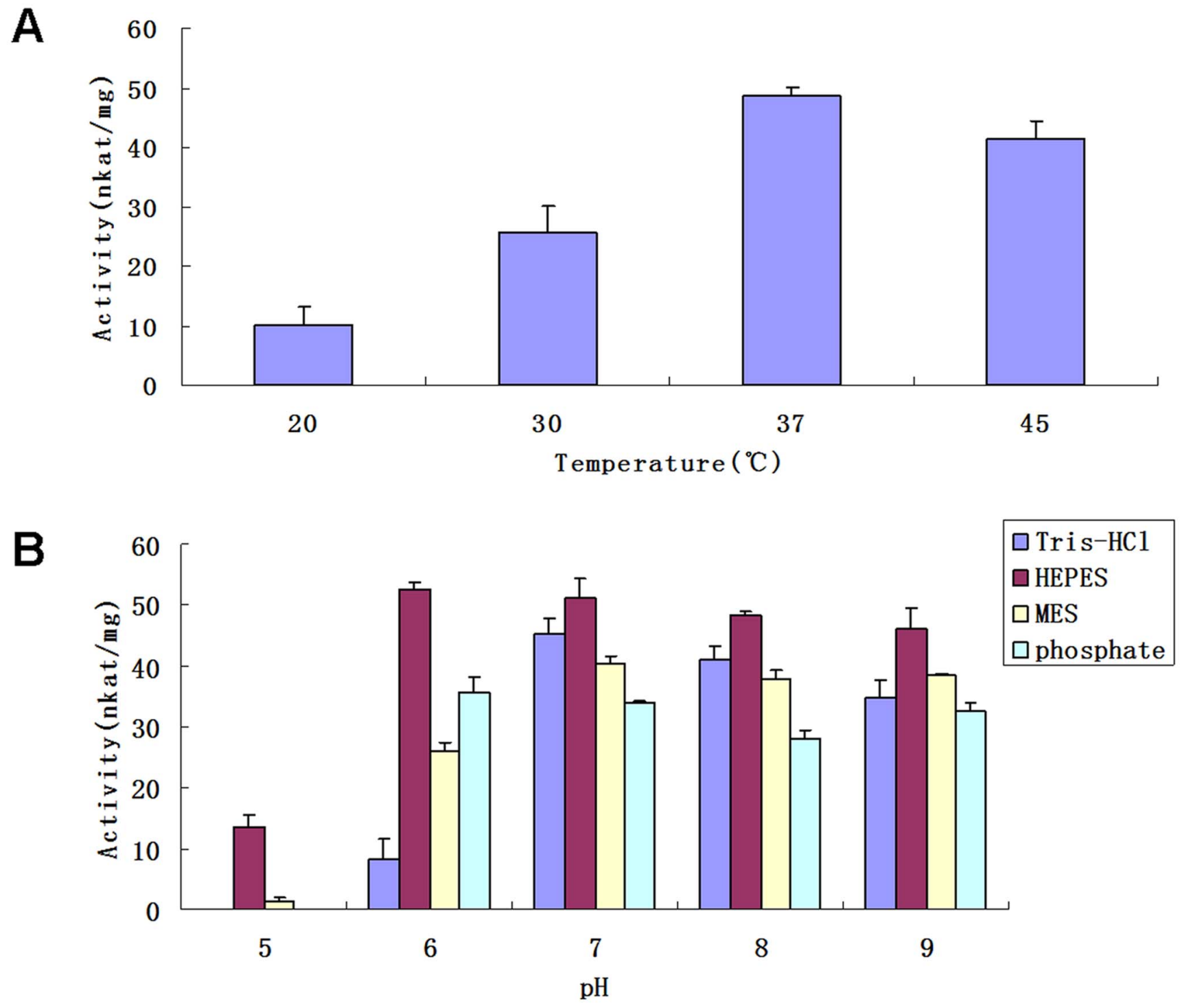

Figure 5. Analyses on factors affecting the activity of recombinant UGT74D1. (A) The effects of temperature. (B) The effects of buffer and $\mathrm{pH}$ value. All the reaction mix $(100 \mu \mathrm{l})$ contained $0.2 \mathrm{ug}$ of recombinant UGT74D1, $5 \mathrm{mM}$ UDP-glucose, $1 \mathrm{mM} \mathrm{IBA,} 2.5 \mathrm{mM} \mathrm{MgSO}$, $10 \mathrm{mM} \mathrm{KCl}$, $14.4 \mathrm{mM}$ 2-mercaptoethanol, $50 \mathrm{mM}$ buffer and was incubated for $30 \mathrm{~min}$ as described in "Materials and Methods". The results represent means \pm SD from three replicates. The specific enzyme activity was defined as nmol of substrates converted into glucose conjugates per second (nanokatal, nkat) by $1 \mathrm{mg}$ of protein.

doi:10.1371/journal.pone.0061705.g005

floral dip method [44]. At least four homozygous transgenic lines were selected by kanamycin resistance and the overexpression of UGT74D1 was determined by RT-PCR.

Total crude protein was extracted from 2-week-old transgenic seedlings as described previously [42]. To investigate the glycosyltransferase activity of the crude protein extracts prepared from plant tissues, $50 \mu \mathrm{l}$ crude protein extracts (containing $\sim 0.1 \mathrm{mg}$ of total protein) were mixed $1 \mathrm{mM}$ auxin, $5 \mathrm{mM}$ UDP-glucose, $50 \mathrm{mM}$ HEPES (pH7.0), $2.5 \mathrm{mM} \mathrm{MgSO} 4,10 \mathrm{mM}$ $\mathrm{KCl}$, and $14.4 \mathrm{mM}$ 2-mercaptoethanol, in a $100 \mu \mathrm{l}$ reaction. The reactions were incubated at $37^{\circ} \mathrm{C}$ for $1 \mathrm{~h}$ and were stopped by the addition of $10 \mu \mathrm{l}$ of trichloroacetic acid $(240 \mathrm{mg} / \mathrm{ml})$. The reaction mix was analyzed subsequently using reverse-phase HPLC following the method described above.

To analyze the amount of the glucose conjugates of interest in the transgenic plants, the wild-type and UGT74D1 transgenic plants (line 23, line 24) were grown on the MS agar plates for 12 days, and removed carefully to immersed in MS liquid culture system with or without $100 \mu \mathrm{M}$ IBA. After incubation for $24 \mathrm{~h}$, $1 \mathrm{~g}$ of plant tissues from each line was collected, frozen in liquid nitrogen, and stored at $-80^{\circ} \mathrm{C}$ prior to the extraction. The extraction of IBA glucose conjugates was carried out following the method described previously [42]. $0.1 \mathrm{mM}$ picloram was added as internal control at the beginning of the extraction to monitor the recovery rate. The amounts of IBA glucose conjugates in extraction buffers of different transgenic lines were analyzed by HPLC as described above.

\section{Leaf-flattening Experiments of Transgenic Plants}

Arabidopsis plants were grown in the greenhouse on Nutrition Soil (Shangdao Biotech Co. Ltd., Shandong, China) with vermiculite (Nutrition Soil:vermiculite $=2: 1$ ) at $22 \pm 2^{\circ} \mathrm{C}$ under a $16 / 8 \mathrm{~h} \mathrm{light} /$ 


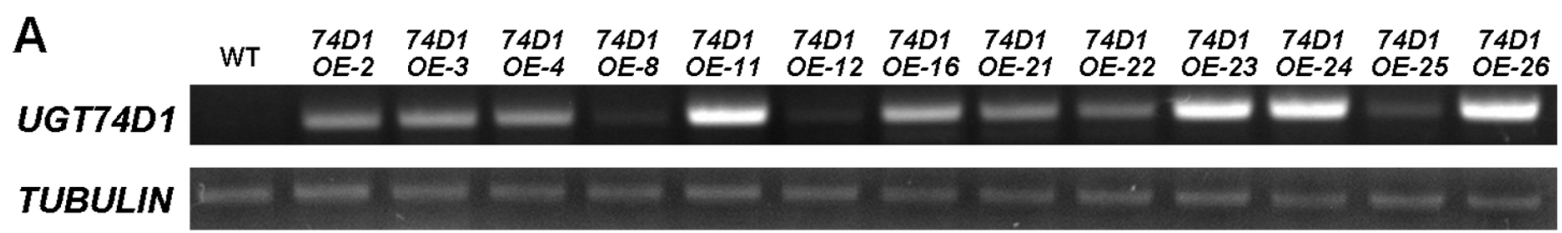

B

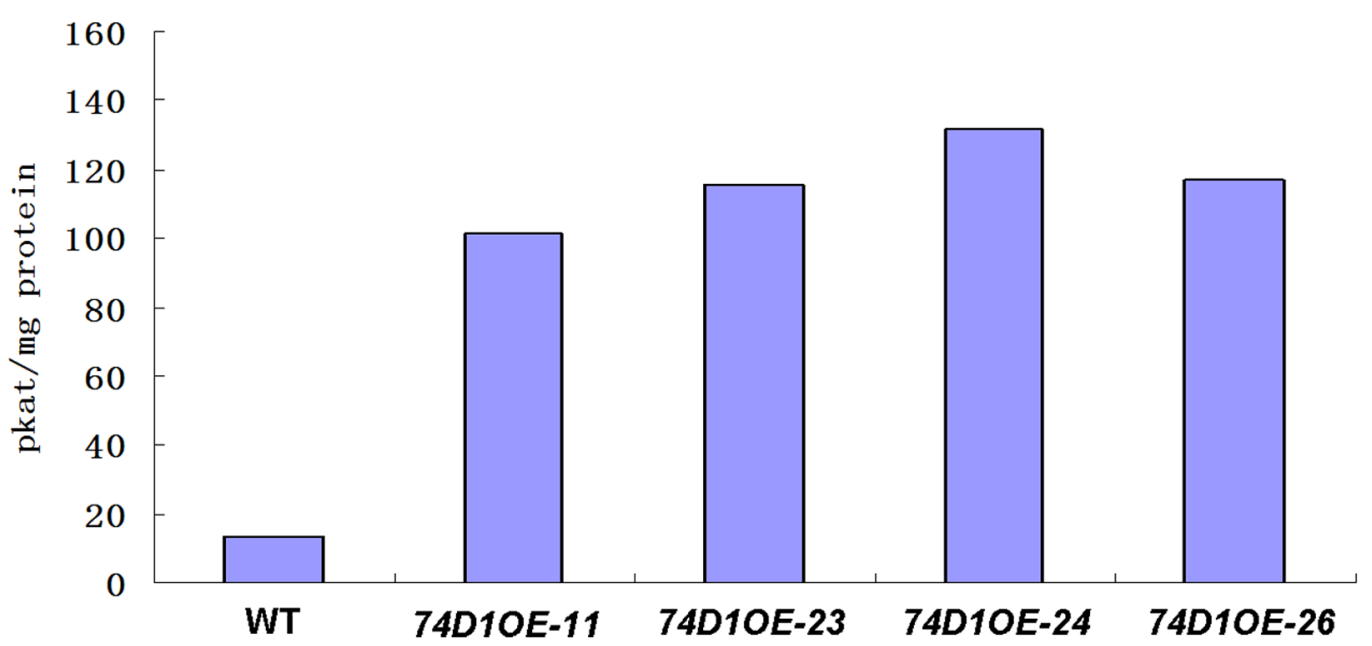

Figure 6. Analysis of the transgenic Arabidopsis plants overexpressing UGT74D1 using the CaMV35S promoter. (A) RT-PCR analyses of the steady-state level of UGT74D1 mRNA in transgenic plants (OEs) and wild type (WT). (B) The glycosyltransferase activities in the crude protein extracts of transgenic plants and wild type were measured following the procedure described under "Materials and Methods". The specific enzyme activity was expressed as pmol of IBA glucosylated to form IBA-Glc by $1 \mathrm{mg}$ of protein per second of reaction time at $37^{\circ} \mathrm{C}$. doi:10.1371/journal.pone.0061705.g006

dark cycle with a light intensity of $\sim 100 \mu \mathrm{mol} \mathrm{m}^{-2} \mathrm{~s}^{-1}$. When plants reached growth stage $\sim 6.5$ after growing for 5 weeks, the lamina of the representative seventh rosette leaf was detached from the petiole and the flattening index was calculated according to the method described by de Carbonnel et al. [45].

\section{Results}

\section{Purification of Recombinant UGT74D1}

In order to explore more hormone-related UGTs, in this study, we put our focus on other members of group L whose activity and substrate have not been previously demonstrated. These UGTs were cloned into prokaryotic expression vector and expressed in Escherichia coli tagged with glutathione $S$-transferase (GST). UGT74D1 gene is predicted to encode a protein of 456 amino acid residues with a theoretical molecular weight of $50.2 \mathrm{kDa}$, thus the recombinant fusion protein should be $76.2 \mathrm{kDa}$ together with GST tag. The SDS-PAGE analysis showed that the molecular mass of the purified fusion protein was between $66.2 \mathrm{kDa}$ and $94.0 \mathrm{kDa}$, which was consistent with the theoretical prediction (Figure 2).
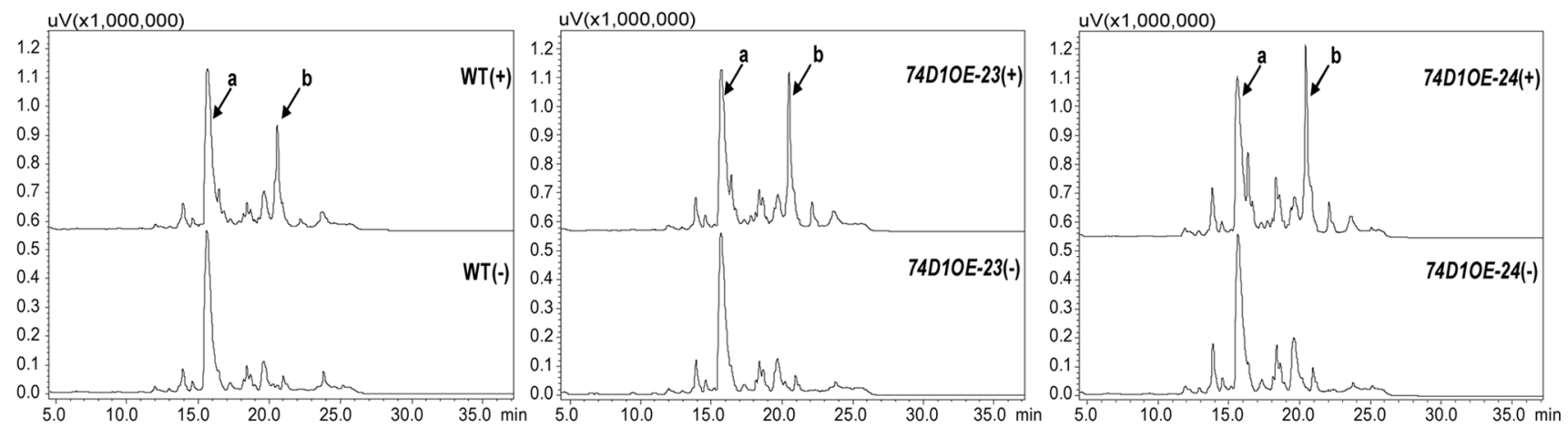

Figure 7. HPLC trace of IBA glucose conjugates of the extracts from the wild type (WT) and transgenic plants (OEs). (+) and (-) represent the plant tissues incubated with or without IBA before extraction process. Peak "a" indicates the picloram added as an internal control at the beginning of the extraction process; Peak " $\mathrm{b}$ " indicates the IBA-glucose conjugates. The extracts were analyzed with a linear gradient of methanol in $\mathrm{H}_{2} \mathrm{O}$ from $10-70 \%$ (all solutions contained $0.01 \% \mathrm{H}_{3} \mathrm{PO}_{4}$ ) over $30 \mathrm{~min}$ at $1 \mathrm{ml} / \mathrm{min}$ and monitored at $280 \mathrm{~nm}$. doi:10.1371/journal.pone.0061705.g007 
Table 2. Phenotype comparison of overexpression lines of UGT74D1, UGT84B1 and UGT74E2 genes.

\begin{tabular}{llll}
\hline & & & \\
\hline Overexpression lines & UGT74D1 & UGT84B1 & UGT74E2 \\
\hline Curling leaf & curling & wrinkly and curling & no \\
Compressed rosette & no & compressed & compressed \\
Shorter stature & no & shorter & shorter \\
Shoot branching & unchanged & higher level & higher level \\
Root gravitropism & unchanged & reduced & unchanged \\
Osmotic stress tolerance & not detected & not detected & increased \\
\hline doi:10.1371/journal.pone.0061705.t002 & &
\end{tabular}

\section{Identification of UGT74D1 Enzymatic Activity Toward Auxins}

Using UDP-glucose as the sugar donor, the recombinant UGT74D1 was tested in vitro for its glycosyltransferase activity against each of the seven substrates used in this study: indole-3carboxylic acid [ICA], indole-3-acetic acid [IAA], indole-3propionic acid [IPA], indole-3-butyric acid [IBA], the synthetic auxin analogs naphthaleneacetic acid [NAA], 2,4-dichlorophenoxyacetic acid [2,4-D], and picloram. The structures of these compounds used are listed in Figure S1. The following HPLC analysis and the recognition of new product peaks showed that the recombinant UGT74Dl had a strong activity toward IBA (Figure 3A). Therefore, we further conducted a LC-MS analysis to the new products. As shown in Figure $3 \mathrm{~B}$, in the positive ionization mode, putative IBA-glucose ester (IBA-Glc) gave a dominant ions $m / z 204.15\left(\mathrm{M}+\mathrm{H}^{+}\right.$-glucose); $m / z 366.16\left(\mathbf{M}+\mathrm{H}^{+}\right)$; $m / z 383.17\left(\mathbf{M}+\mathrm{NH}_{4}{ }^{+}\right)$and $m / z 388.12\left(\mathrm{M}+\mathrm{Na}^{+}\right)$(MW of IBA-Glc is 365.00). The mass spectrum peaks of putative IBA-Glc were identical to the peaks of a product catalyzed by UGT74E2 [23], which was demonstrated to be IBA-Glc and used as a positive control in our research (Figure 3C). Thus, a new biosynthetic pathway of IBA-glucose ester from the aglycone IBA by UGT74D1 catalysis was proposed (Figure 3D).

As shown in Figure 4, UGT74D1 also had a significant activity toward other auxins with similar structure to IBA, for example, IPA, IAA and NAA, only a trace activity toward 2,4-D and ICA, whereas no activity toward picloram. The specific enzyme activities of UGT74D1 towards different substrates were also calculated (Table 1), and the data indicated that UGT74D1 was an auxin glycosyltransferase with the highest activity towards IBA.

The retention time $(\mathrm{Rt})$ and $\lambda_{\max }$ of the glucose conjugates produced were as follows: ICA conjugate, $\mathrm{Rt}=14.2 \mathrm{~min}$, $\lambda_{\text {max }}=280 \mathrm{~nm} ;$ IAA conjugate, $\mathrm{Rt}=22.0 \mathrm{~min}, \lambda_{\max }=210 \mathrm{~nm}$; IPA conjugate, $R t=19.7 \mathrm{~min}, \lambda_{\max }=280 \mathrm{~nm}$; IBA conjugate, $\mathrm{Rt}=21.3 \mathrm{~min}, \lambda_{\max }=280 \mathrm{~nm} ; \mathrm{NAA}$ conjugate, $\mathrm{Rt}=21.7 \mathrm{~min}$, $\lambda_{\text {max }}=280 \mathrm{~nm} ; 2,4-\mathrm{D}$ conjugate, $\mathrm{Rt}=24.0 \mathrm{~min}, \lambda_{\max }=287 \mathrm{~nm}$.

\section{Biochemical Characterization of UGT74D1}

The results in Figure 5 summarize the effects of reaction conditions, including temperature, $\mathrm{pH}$ and buffer, on the catalytic activity of UGT74D1. Four temperature points were tested and the results showed that $37^{\circ} \mathrm{C}$ was the best (Figure 5A). The pH analysis using Tris-HCl buffer, HEPES buffer, MES buffer, and phosphate buffer showed that UGT74D1 was active over a broad $\mathrm{pH}$ range but with a maximum in HEPES buffer at $\mathrm{pH} 6.0$ (Figure 5B).
Enzyme Activity Analysis of Transgenic Arabidopsis Plants Overexpressing UGT74D1

To gain further insights into the UGT74Dl activity, the transgenic plants overexpressing UGT74D1 driven by cauliflower mosaic virus 35S (CaMV35S) promoter were generated, and ten independent homozygous lines were obtained. As shown in Figure 6A, higher steady-state UGT74D1 level was observed in transgenic lines than that in wild-type plants. Seedlings of four transgenic lines were analyzed for enzyme activity toward IBA (Figure 6B). The results demonstrated that lines with higher UGT74D1 transcripts also displayed stronger enzyme activity than wild type to form IBA-glucose conjugates.

\section{Glucosylated Metabolite Analysis of Transgenic Arabidopsis Plants}

To see whether the glucosidic metabolite is altered by enhanced expression of UGT74D1, exogenous IBA is applied to the transgenic and WT plants. As shown in Figure 7, if the plant tissues were not incubated with IBA before extraction process, IBA-glucose conjugates were below the level that could be detected or reliably quantified in our HPLC analysis. Upon application of IBA, however, considerable level of IBA-glucose conjugates were observed in both WT and transgenic plants. Compared to the amount of IBA-glucose produced in WT plants (130.81 pmol/mg.FW), much higher level of IBA-glucose in transgenic lines 74DlOE-23 (200.51 pmol $/ \mathrm{mg} . \mathrm{FW})$ and 74D1OE-24 (277.55 pmol/mg.FW) can be detected. These data indicated that over-production of the UGT74D1 in the plants indeed led to increased level of the glucose conjugate of IBA.

\section{Phenotypes of Transgenic Arabidopsis Plants}

Two knockout mutants, 74d1ko-1 (Salk_004870) and 74d1ko-2 (Salk_011286), were confirmed to have no UGT74D1 transcripts (data not shown). Two transgenic lines over-expressing UGT74D1, 74D1OE-23 and 74D1OE-24, were also confirmed (Figure 6). Preliminary observation indicated that, although homozygous knockout plants and overexpression lines had the similar phenotypes with wild-type including shoot height, shoot branching and root gravitropism (Figure 8A, Table 2), UGT74D1OE plants displayed curling leaves that differed from those of the wild-type plants at flowering stage (Figure 8B, 8C, 8D). The curling leaf phenotype of UGT74D1OE plants began to emerge after growing for four weeks, but was even more pronounced after growing for five weeks (growth stage $\sim 6.5$ ), suggesting a physiological role of UGT74D1 in affecting the activity of auxins in leaves at this developmental stage.

\section{Discussion}

Glycosylation is a widespread physiological phenomenon, and is thought to be one of the most important mechanisms in maintaining plant cell homeostasis [34]. Glycosyltransferases are the enzymes responsible for glycosylation. They can typically transfer single or multiple activated sugars from nucleotide sugar donors, especially UDP-glucose, to a wide range of small molecular acceptors, thus change their bioactivity, solubility, stability, subcellular localization and binding properties. A detailed phylogenetic analysis classified the Arabidopsis familyl glycosyltransferases into 14 groups $(\mathrm{A}-\mathrm{N})$ based on their sequence homology and pattern of intron gain [39]. Several members of group L have been identified to glucosylate plant compounds to form their glucose esters [23,37,46-48]. In this study, we provide solid evidence that UGT74D1 of group L is a novel glycosyltransferase that can catalyze auxin glycosylation. Our results lay 


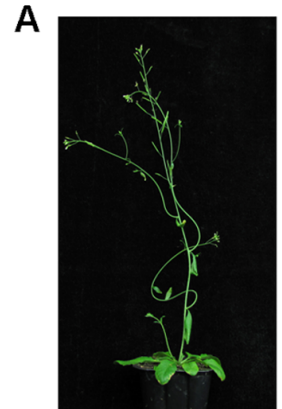

WT

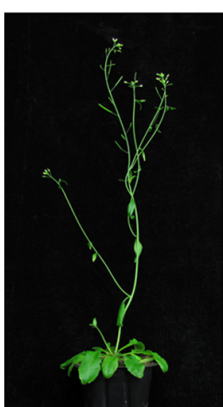

74d1ko-1

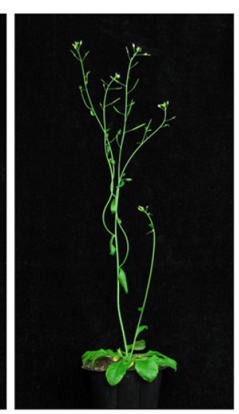

74d1ko-2

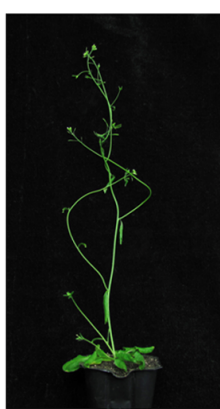

74D1OE-23

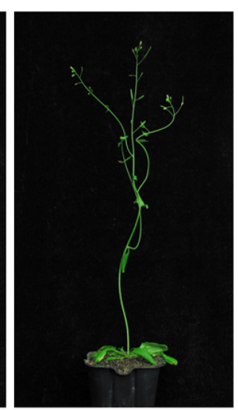

74D1OE-24

B

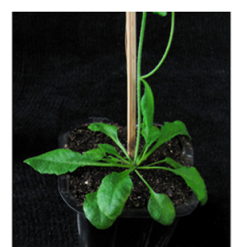

WT

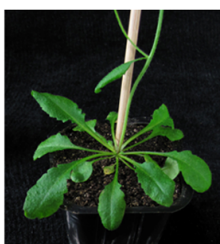

74d1ko-1

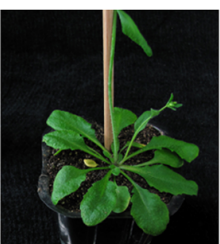

74d1ko-2

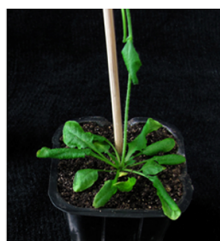

74D1OE-23

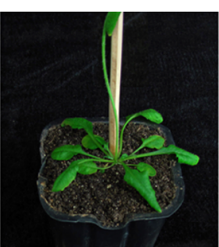

$74 D 1 O E-24$

C

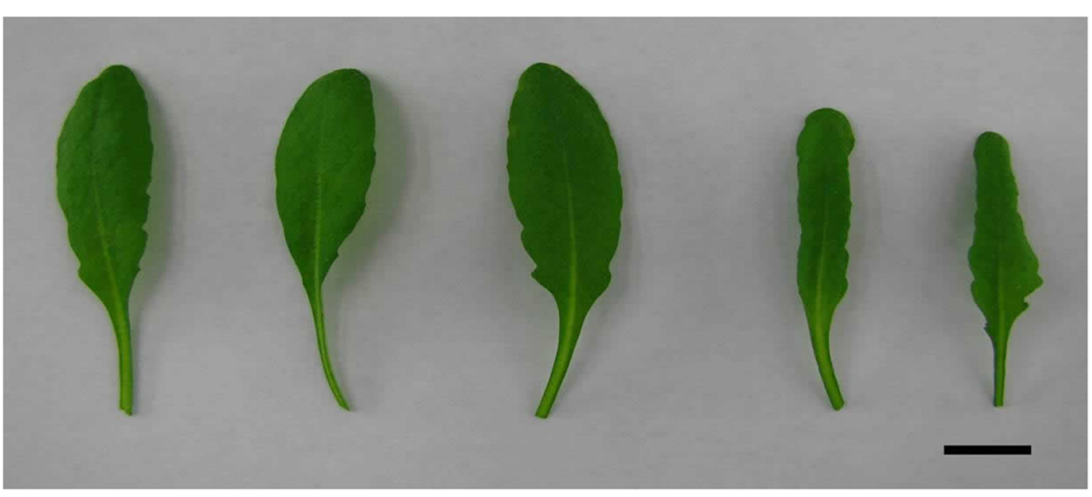

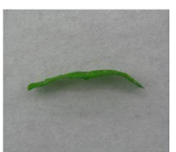

WT

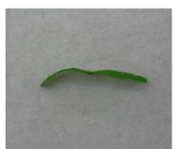

74d1ko-1

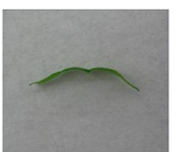

74d1ko-2

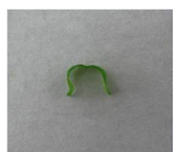

74D1OE-23

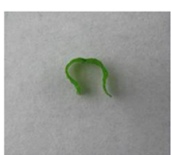

74D1OE-24

D

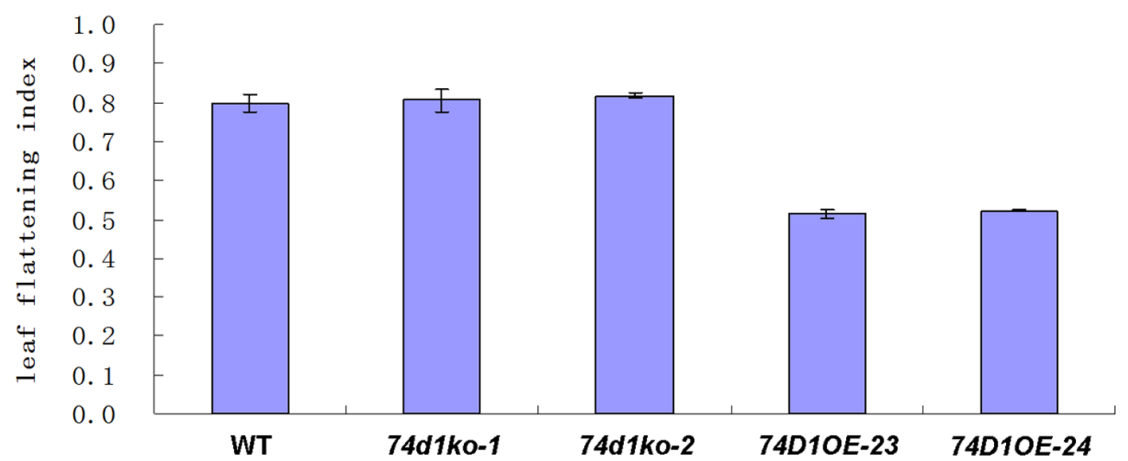

Figure 8. Phenotypes of Transgenic Arabidopsis Plants. (A) 5-week-old plant phenotypes of WT, mutants and overexpression lines. (B) 5-weekold rosette leaf phenotypes of wild type, mutants and overexpressor lines. (C) Seventh leaf and leaf transverse section of 5-week-old plants. (D) The flattening index of seventh leaf was calculated by dividing the projection area of intact curled leaves with that of manually uncurled leaves. doi:10.1371/journal.pone.0061705.g008 
the groundwork for future genetic approaches in better understanding the regulation of auxin homeostasis by glycosylation in plants.

The data present here showed that UGT74D1 had different enzyme activities toward different auxins: IBA $>$ IPA $>$ IAA $>$ "' NAA $>2,4-D>$ ICA. It appears that the substrate preference of UGT74D 1 might result from its regioselectivity to substrates and the side chain length of auxins which plays a major role in determining the glucosylating activity, thus the highest activity of UGT74D1 is with IBA and the lowest is with ICA. As yet, the relationship of these activities to events within the plants is unknown. Although IBA is the preferred substrate for UGT74D1 in vitro, the enzyme may glucosylate both IAA and IBA in planta depending on cell specificity of the enzyme expression, relative availability of substrates, and relative compartmentation of the enzyme and substrates.

Up to now, there has been several master glycosyltransferases identified from Arabidopsis to be responsible for the auxin glycosylation which include UGT84B1 mainly toward IAA [37], UGT74E2 and UGT74D1 mainly toward IBA [23, and this research]. These findings suggest that plant evolution has involved the formation of functionally redundant multiple glycosyltransferases toward the same type of phytohormones. It is therefore possible that additional glycosyltransferases may exist in Arabidopsis that are capable of glucosylating auxins. Why do functionally redundant auxin glycosyltransferases exist in plants? A synergistic or coordinated effect between different glycosyltransferase members may be meaningful for the fine tuning of auxin homeostasis. On the other hand, the spatial-temporal expression patterns of these genes might be different, which may have the potential to enhance the plant flexibility in development or in the adaptation to diverse environments.

It was reported that the constitutive expression of UGT84B1 or UGT74E2 in Arabidopsis resulted in many features typical of auxin-

\section{References}

1. Woodward AW, Bartel B (2005) Auxin: regulation, action, and interaction. Ann Bot 95: 707-735.

2. Benjamins R, Scheres B (2008) Auxin: the looping star in plant development. Annu Rev Plant Biol 59: 443-465.

3. Vanneste S, Friml J (2009) Auxin: a trigger for change in plant development. Cell 136: 1005-1016.

4. Kazan K, Manners JM (2009) Linking development to defense: auxin in plantpathogen interactions. Trends Plant Sci 14: 373-382.

5. Kieffer M, Neve J, Kepinski S (2010) Defining auxin response contexts in plant development. Curr Opin Plant Biol 13: 12-20.

6. Cohen JD, Bandurski RS (1982) Chemistry and Physiology of the Bound Auxins. Annu Rev Plant Physiol 33: 403-430.

7. Ludwig-Müller J, Sass S, Sutter EG, Wodner M, Epstein E (1993) Indole-3butyric acid in Arabidposis thaliana. I. Identification and quantification. Plant Growth Regul 13: 179-187.

8. Ludwig-Müller J (2000) Indole-3-butyric acid in plant growth and development. Plant Growth Regul 32: 219-230.

9. Ludwig-Müller J, Epstein E (1993) Indole-3-butyric acid in Arabidopsis thaliana. II. In vivo metabolism. Plant Growth Regul 13: 189-195.

10. Bartel B, LeClere S, Magidin M, Zolman BK (2001) Inputs to the active indole3-acetic acid pool: De novo synthesis, conjugate hydrolysis, and indole-3-butyric acid $\beta$-oxidation. J Plant Growth Regul 20: 198-216.

11. Zolman BK, Silva ID, Bartel B (2001a) The Arabidopsis pxal mutant is defective in an ATP-binding cassette transporter-like protein required for peroxisomal fatty acid b-oxidation. Plant Physiol 127: 1266-1278.

12. Zolman BK, Monroe-Augustus M, Thompson B, Hawes JW, Krukenberg KA, et al. (2001b) chyl, an Arabidopsis mutant with impaired $\beta$-oxidation, is defective in a peroxisomal b-hydroxyisobutyryl-CoA hydrolase. J Biol Chem 276: 31037-31046.

13. Zolman BK, Nyberg M, Bartel B (2007) IBR3, a novel peroxisomal acyl-CoA dehydrogenase-like protein required for indole-3-butyric acid response. Plant Mol Biol 64: 59-72.

14. Zolman BK, Martinez N, Millius A, Adham AR, Bartel B (2008) Identification and characterization of Arabidopsis indole-3-butyric acid response mutants defective in novel peroxisomal enzymes. Genetics 180: 237-251. deficient phenotypes [23,38]. These findings indicated the significance of those auxin glycosyltransferases in maintaining normal growth and development of plants. However, it appears that those auxin glycosyltransferases do not have exactly the same role. For example, UGT84B1 and UGT74E2 overexpresssors displayed the same phenotypes in compressed rosette, shorter stature and more shoot branches. On the other hand, UGT84B1 overexpressors also had wrinkle leaves and reduced root gravitropism, but UGT74E2 overexpresssors don't (Table 2). Our observations in this present study on transgenic lines indicate that UGT74D1 has an influence on leaf growth, resulting in curling leaves of transgenic plants, but no other phenotypes were observed. Thus, the data described suggest that UGT74D1 is a novel auxin-UGT and has specific effects, providing a new target gene for further genetic study of auxin activity and regulation. Through further analyses of cell- and environment-specific expression of UGT74D1, followed by detailed metabolite profiling of auxins, we would get more insights into its in vivo substrates, its physiological impact on auxin homeostasis and even its possible synergistic effect with other auxin glycosyltransferases.

\section{Supporting Information}

Figure S1 The molecular structures of auxins used in this study as substrates for the enzymatic activity identification of UGT74D1.

(TIF)

\section{Author Contributions}

Conceived and designed the experiments: BKH. Performed the experiments: SHJ XMM PH. Analyzed the data: SHJ BW YGS. Contributed reagents/materials/analysis tools: SHJ GZZ YJL. Wrote the paper: BKH YJL SHJ
15. Strader LC, Culler AH, Cohen JD, Bartel B (2010) Conversion of endogenous indole-3-butyric acid to indole-3-acetic acid drives cell expansion in Arabidopsis seedlings. Plant Physiol 153: 1577-1586.

16. Strader LC, Wheeler DL, Christensen SE, Berens JC, Cohen JD, et al. (2011) Multiple facets of Arabidopsis seedling development require indole-3-butyric acidderived auxin. Plant Cell 23: 984-999.

17. Strader LC, Bartel B (2011) Transport and metabolism of the endogenous auxin precursor indole-3-butyric acid. Mol Plant 4: 477-486.

18. Zolman BK, Yoder A, Bartel B (2000) Genetic analysis of indole-3-butyric acid responses in Arabidopsis thaliana reveals four mutant classes. Genetics 156: 1323-1337.

19. Rashotte AM, Poupart J, Waddell CS, Muday GK. (2003) Transport of the two natural auxins, indole-3-butyric acid and indole-3-acetic acid, in Arabidopsis. Plant Physiol 133: 761-772.

20. Ludwig-Muller J, Hilgenberg W (1995) Characterization and partial purification of indole-3-butyric acid synthetase from maize (Zea mays). Physiol Plant 94: 651-660.

21. Oono Y, Chen QG, Overvoorde PJ, Kohler C, Theologis A (1998) age Mutants of Arabidopsis exhibit altered auxin-regulated gene expression. Plant Cell 10: $1649-1662$.

22. Ulmasov T, Hagen G, Guilfoyle TJ (1999) Activation and repression of transcription by auxin-response factors. Proc Natl Acad Sci USA 96: 5844-5849.

23. Tognetti VB, Van Aken O, Morreel K, Vandenbroucke K, van de Cotte B, et al. (2010) Perturbation of indole-3-butyric acid homeostasis by the UDPglucosyltransferase UGT74E2 modulates Arabidopsis architecture and water stress tolerance. Plant Cell 22: 2660-2679.

24. Poupart J, Rashotte AM, Muday GK, Waddell CS (2005) The rib1 mutant of Arabidopsis has alterations in indole-3-butyric acid transport, hypocotyl elongation, and root architecture. Plant Physiol 139: 1460-1471.

25. Liu X, Barkawi L, Gardner G, Cohen JD (2012) Transport of indole-3-butyric acid and indole-3-acetic acid in arabidopsis hypocotyls using stable isotope labeling. Plant Physiol 158: 1988-2000.

26. Bajguz A, Piotrowska A (2009) Conjugates of auxin and cytokinin. Phytochemistry 70: 957-969. 
27. Ljung K, Hull AK, Kowalczyk M, Marchant A, Celenza J, et al. (2002) Biosynthesis, conjugation, catabolism and homeostasis of indole-3-acetic acid in Arabidopsis thaliana. Plant Mol Biol 49: 249-272.

28. Park JE, Park JY, Kim YS, Staswick PE, Jeon J, et al. (2007) GH3-mediated auxin homeostasis links growth regulation with stress adaptation response in Arabidopsis. J Biol Chem 282: 10036-10046.

29. Ludwig-Muller J (2011) Auxin conjugates: their role for plant development and in the evolution of land plants. J Exp Bot 62: 1757-73.

30. Bandurski RS, Cohen JD, Slovin JP, Reinecke DM (1995) Auxin biosynthesis and metabolism. In Plant Hormones, P.J. Davies, ed (Dordrecht, The Netherlands: Kluwer Academic Publishers), 118-139.

31. Epstein E, Ludwig-Müller J (1993) Indole-3-butyric acid in plants: occurrence, synthesis, metabolism and transport. Physiol Plantarum 88: 382-389.

32. Rampey RA, LeClere S, Kowalczyk M, Ljung K, Sandberg G, et al. (2004) A family of auxin-conjugate hydrolases that contributes to free indole-3-acetic acid level during Arabidopsis germination. Plant Physiol 135: 978-988.

33. Tam YY, Epstein E, Normanly J (2000) Characterization of auxin conjugates in Arabidopsis. Low steady-state level of indole-3-acetyl-aspartate, indole-3-acetylglutamate, and indole-3-acetyl-glucose. Plant Physiol 123: 589-595.

34. Lim EK, Bowles DJ (2004) A class of plant glycosyltransferases involved in cellular homeostasis. EMBO J 23: 2915-2922.

35. Bowles D, Lim EK, Poppenberger B, Vaistij FE (2006) Glycosyltransferases of lipophilic small molecules. Annu Rev Plant Biol 57: 567-597.

36. Szerszen JB, Szczyglowski K, Bandurski RS (1994) iaglu, a gene from Zea mays involved in conjugation of growth hormone indole-3-acetic acid. Science 265: 1699-1701.

37. Jackson RG, Lim EK, Li Y, Kowalczyk M, Sandberg G, et al. (2001) Identification and biochemical characterization of an Arabidopsis indole-3-acetic acid glucosyltransferase. J Biol Chem 276: 4350-4356.
38. Jackson RG, Kowalczyk M, Li Y, Higgins G, Ross J, et al. (2002) Overexpression of an Arabidopsis gene encoding a glucosyltransferase of indole-3-acetic acid: phenotypic characterisation of transgenic lines. Plant J 32: 573-583.

39. Ross J, Li Y, Lim EK, Bowles DJ (2001) Higher plant glycosyltransferases. Genome Biol 2: reviews3004.1-3004.6.

40. Li Y, Baldauf S, Lim EK, Bowles DJ (2001) Phylogenetic analysis of the UDPglycosyltransferase multigene family of Arabidopsis thaliana. J Biol Chem 276: 4338-4343.

41. Zhang H, Ohyama K, Boudet J, Chen Z, Yang J, et al. (2008) Dolichol biosynthesis and its effects on the unfolded protein response and abiotic stress resistance in Arabidopsis. Plant Cell 20: 1879-1898.

42. Hou B, Lim EK, Higgins GS, Bowles DJ (2004) N-glucosylation of cytokinins by glycosyltransferases of Arabidopsis thaliana. J Biol Chem 279: 47822-47832.

43. Sambrook J, Fritsch EF, Maniatis T (1989) Molecular Cloning: A Laboratory Manual, 2nd edn. Cold Spring Harbor, New York: Cold Spring Laboratory Press.

44. Clough SJ, Bent AF (1998) Floral dip: a simplified method for Agrobacteriummediated transformation of Arabidopsis thaliana. Plant J 16: 735-743.

45. de Carbonnel M, Davis P, Roelfsema MRG, Inoue S, Schepens I, et al. (2010) The Arabidopsis PHYTOCHROME KINASE SUBSTRATE2 protein is a phototropin signaling element that regulates leaf flattening and leaf positioning. Plant Physiol 152: 1391-1405.

46. Grubb CD, Zipp BJ, Ludwig-Müller J, Masuno MN, Molinski TF, et al. (2004) Arabidopsis glucosyltransferase UGT74B1 functions in glucosinolate biosynthesis and auxin homeostasis. Plant J 40: 893-908.

47. Lim EK, Doucet CJ, Li Y, Elias L, Worrall D, et al. (2002) The activity of Arabidopsis glycosyltransferases toward salicylic acid, 4-hydroxybenzoic acid, and other benzoates. J Biol Chem 277: 586-592.

48. Lim EK, Li Y, Parr A, Jackson R, Ashford DA, et al. (2001) Identification of glucosyltransferase genes involved in sinapate metabolism and lignin synthesis in Arabidopsis. J Biol Chem 276: 4344-4349. 